\title{
ADAPTIVE MULTI-OBJECTIVE SUB-PIXEL MAPPING FRAMEWORK BASED ON MEMETIC ALGORITHM FOR HYPERSPECTRAL REMOTE SENSING IMAGERY
}

\author{
Yanfei Zhong ${ }^{\text {a, } * \text {, Liangpei Zhang }}{ }^{\text {a }}$ \\ a State Key Laboratory of information Engineering in Surveying Mapping and Remote Sensing, Wuhan University, 129 Luoyu \\ Road, Wuhan, Hubei province, 430079, China-zhongyanfei@lmars.whu.edu.cn
}

\section{Commission VII/4}

KEY WORDS: Hyperspectral, Sub-pixel mapping, Multi-objective, Memetic algorithm, Artificial Intelligence, Remote sensing

\begin{abstract}
:
Sub-pixel mapping technique can specify the location of each class within the pixels based on the assumption of spatial dependence. Traditional sub-pixel mapping algorithms only consider the spatial dependence at the pixel level. The spatial dependence of each sub-pixel is ignored and sub-pixel spatial relation is lost. In this paper, a novel multi-objective sub-pixel mapping framework based on memetic algorithm, namely MSMF, is proposed. In MSMF, the sub-pixel mapping is transformed to a multi-objective optimization problem, which maximizing the spatial dependence index (SDI) and Moran's I, synchronously. Memetic algorithm is utilized to solve the multi-objective problem, which combines global search strategies with local search heuristics. In this framework, the sub-pixel mapping problem can be solved using different evolutionary algorithms and local algorithms. In this paper, memetic algorithm based on clonal selection algorithm (CSA) and random swapping as an example is designed and applied simultaneously in the proposed MSMF. In MSMF, CSA inherits the biologic properties of human immune systems, i.e. clone, mutation, memory, to search the possible sub-pixel mapping solution in the global space. After the exploration based on CSA, the local search based on random swapping is employed to dynamically decide which neighbourhood should be selected to stress exploitation in each generation. In addition, a solution set is used in MSMF to hold and update the obtained non-dominated solutions for multi-objective problem. Experimental results demonstrate that the proposed approach outperform traditional sub-pixel mapping algorithms, and hence provide an effective option for sub-pixel mapping of hyperspectral remote sensing imagery.
\end{abstract}

\section{INTRODUCTION}

The mixed pixel is a common phenomenon in remote sensing image because the sensor's instantaneous field-of-view (IFOV) often includes more than one land cover class on the ground (Chang, 2003). The Land-cover classification accuracy may be severely compromised by the presence of mixed pixels. Although spectral unmixing technique (Chang, 2003) and fuzzy classifiers can obtain the fraction image indicating the percentage of each class in the mixed pixel, they do not provide any indication about the sub-pixel spatial distribution of the classes within the coarse pixel. Sub-pixel mapping techniques can be used to specify the sub-pixel spatial distribution of each class, based on the fraction image, by dividing pixels into smaller sub-pixels based on the spatial dependence phenomenon (Atkinson, 1997) in which observations close together are more alike than those further apart. The key problem of sub-pixel mapping is determining the most likely locations of the fractions of each land cover class within the pixel (Verhoeye and Wulf, 2002).

Different potential techniques have been proposed for sub-pixel mapping based on the assumption of the spatial dependence, such as sub-pixel mapping algorithms based on direct neighboring algorithm (DNSM), spatial attraction model (SASM) (Mertens et al., 2006), BP neural network (BPSM) (Zhang, et al., 2008), Linear optimization techniques (Verhoeye and Wulf, 2002), Genetic algorithm (GASM) (Mertens et al., 2003). These current sub-pixel mapping algorithms may obtain the classification result with a finer resolution, but they only consider the spatial dependence at the pixel level by utilizing the neighbour pixels of the mixed pixel. These sub-pixel mapping algorithms with calculation at pixel level ignore the spatial dependence of each sub-pixel and they can be further improved by using the spatial information at sub-pixel level.

In this paper, to consider the spatial dependence between pixels and sub-pixels together, a new search strategy inspired by multi-objective memetic algorithm (Ong et al., 2010), namely multi-objective memetic sub-pixel mapping framework (MSMF), is proposed. In MSMF, the sub-pixel mapping problem becomes a multi-objective optimization problem, which one of objective function using spatial dependence index (SDI) describes the spatial dependence between pixels (Verhoeye and Wulf, 2002), and another function with Moran's I considers the spatial dependence between sub-pixels (Moran, 1950). Memetic algorithm (Ong et al., 2010), a union of a population-based global search and local improvements, provides a power and effective algorithm for multi-objective optimization problem. MSMF utilizes memetic algorithm to construct a multi-objective sub-pixel mapping optimization framework with global and local search. In this opening framework, global search may be carried out using different evolutionary algorithms, such as artificial immune systems (Dasgupta et al., 2011), differential evolution (Zhong and Zhang, 2012). Local search for sub-pixel mapping may be designed by random algorithm or pixel swapping algorithm. In this paper, MSMF utilizes artificial immune systems for global search, and random algorithm for local search to perform the task of the sub-pixel mapping for hyperspectral remote sensing imagery as follows. (1) In MSMF, each candidate individual represents the possible sub-pixel configuration of the pixel, and Pareto dominated individuals based upon multi-objective function evaluations are removed by Pareto ranking. (2)

\footnotetext{
* Corresponding author.
} 
Evolutionary operations are performed to generate new individuals by the adaptive evolution operators, such as clonal, selection, mutation. These operators can draw the evolutionary process closer to the goal, i.e. the optimal sub-pixel distribution and these parameters can be adaptively calculated without userdefined. For example, the mutation rate is determined according to the value of objective function, which better individual will have smaller mutation rate. (3) After evolution, Pareto dominated and infeasible individuals are removed and good individuals are retained for the next generation by using ranking and elitism selection. (4) In addition, to avoid stagnation in global search, MSMF explores the local neighbourhood regions in objective space to find a more feasible solution. The proposed method was tested using the synthetic and degraded real imagery, and experimental results demonstrate that the proposed approach has better results.

The remainder of this paper is organized as follows. Section 2 provides the mathematical formulation of the sub-pixel mapping algorithm, and Section 3 deals with the multi-objective memetic algorithm. Section 4 presents the proposed adaptive multi-objective sub-pixel mapping framework based on memetic algorithm for remote sensing imagery, namely MSMF. In Section 5, the experimental results are provided. Finally, the conclusion is given in Section 6.

\section{MULTI-OBJECTIVE OPTIMIZATION PROBLEM DEFINITION FOR SUB-PIXEL MAPPING}

Sub-pixel mapping is a technique designed to specify the assumption of spatial dependence. The assumption is based on the tendency for spatially proximate observations of a given property to be more alike than more distant observations. The basic principle of sub-pixel mapping is illustrated in Figure 1 by a simple example with two classes (class 1 and class 2), which each pixels is divided into $4(2 \times 2)$ sub-pixels. The fraction image of land-cover class 1 is shown in Figure 1 (a), and the proportion of another class, class 2, may be obtained by subtracting 1 from the fraction of the corresponding class 1 in the same pixel. Figure 2 (b) and Figure 2 (c) are two possible sub-pixel mapping solutions for Figure 2 (a). According to the assumption of spatial dependence, the solution of Figure 2 (b) is better than Figure 2 (c)

\begin{tabular}{|l|l|l|}
\hline 0.5 & 0.25 & 0 \\
\hline 1.0 & 0.5 & 0 \\
\hline 0.5 & 0 & 0 \\
\hline
\end{tabular}

(a) The fraction image of class $1(3 \times 3)$

class 1

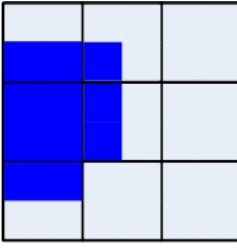

(b) A possible result $(6 \times 6)$

class 2

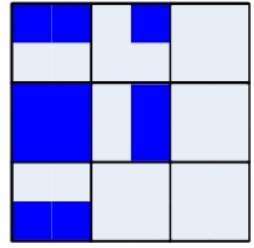

(c) Another possible result $(6 \times 6)$
Figure 1. An example of sub-pixel mapping ( $3 \times 3$ sub-pixels)

It is assumed that the land cover is spatially dependent between pixels and sub-pixels. The sub-pixel mapping problem needs the spatial dependence model between pixels and sub-pixels, respectively, to determine the most likely locations of the fractions of each land cover class. In this paper, the spatial dependence index (SDI) and Moran's I are utilized to evaluate the spatial dependence of the sub-pixel mapping result between pixels and sub-pixels. Without the loss of generality, sub-pixel mapping can be formulated a multi-objective optimization problem.

$$
\begin{aligned}
& \text { Max } Y=f(X)=\left(f_{1}(X), f_{2}(X), \cdots, f_{m}(X)\right) \text {, } \\
& \text { s.t. } g(X)=\left(g_{1}(X), g_{2}(X), \cdots, g_{k}(X)\right)
\end{aligned}
$$

where the decision vector $X=\left(x_{1}, x_{2}, \cdots, x_{n}\right) \in \Omega$, the objective vector $Y \in \Lambda, g_{i}(x)$ is the constrain condition, $\Omega$ denotes the decision space, $\Lambda$ is called as the objective space.

\subsection{Spatial dependence Index (SDI)}

Let us suppose the linear pixel unmixing model yields the fraction images for $C$ land cover classes and the coarse resolution pixels are to be divided into $D$ sub-pixels. To construct the mathematical model, the attribute of each subpixel can be defined by $x_{i j}$ as follows

$$
x_{i j}=\left\{\begin{array}{l}
1, \text { if } \text { sub }- \text { pixel } \mathrm{j} \text { is assigned to land cover class } \mathrm{i} \\
0, \text { otherwise }
\end{array}\right.
$$

where $j=1,2, \ldots, D, i=1,2, \cdots, C$. The spatial dependence mathematical model $z$ is computed by the following equation (Verhoeye and Wulf, 2002):

$$
\begin{aligned}
& \text { Maximize } f_{1}(x)=z=\sum_{i=1}^{C} \sum_{j=1}^{D} x_{i j} * S D I_{i j} \\
& \text { s.t. } \sum_{i=1}^{C} x_{i j}=1 \quad \sum_{j=1}^{D} x_{i j}=N C_{i}
\end{aligned}
$$

where $N C_{i}$ is the number of sub-pixels that have to assigned to land cover class $i$.

The method uses fraction values of neighboring pixels to find the sub-pixel location of the fractions inside the pixel under consideration. The neighboring pixels are considered to have an influence attracting the sub-pixels of the same class in neighboring pixels. So, $S D I_{i j}$ can be calculated as follows:

$$
S D I_{i j}=\sum_{k=1}^{N} w_{k}^{*} \text { Fraction }_{k}
$$

where $w_{k}$ is the weight of each neighboring pixel, $N$ is the number of neighboring pixels, Fraction ${ }_{k}$ is the fraction of the $k$ th neighboring pixel for the $i$-th land cover class. Thus, the sub-pixel mapping problem of $D$ sub-pixels with $C$ land cover classes can be suitably formulated as an optimization problem to find the global maximum of the criterion function $z$ (See also equation (3)).

\subsection{Moran's I}

In statistics, Moran's I is a measure of spatial autocorrelation developed by Moran (Moran, 1950). Spatial autocorrelation is characterized by a correlation in a signal among nearby locations in space. So, in this paper, Moran's I is used to estimate the spatial dependence between sub-pixels as follows. 


$$
\text { Maximize } f_{2}(x)=I=\frac{N_{A} \sum_{i} \sum_{j} w_{i j}\left(x_{i}-\bar{x}\right)\left(x_{j}-\bar{x}\right)}{\left(\sum_{i} \sum_{j} w_{i j}\right) \sum_{i}\left(x_{i}-\bar{x}\right)^{2}}
$$

where $N_{A}$ is the number of cases, $x_{i}$ is the variable value at a particular location, $x_{j}$ is the variable value at another location, $\bar{x}$ is the mean of the variable, and $w_{i j}$ is a weight applied to the comparison between location $i$ and location $j$.

Negative (positive) values indicate negative (positive) spatial autocorrelation. The value of Moran's I varies between -1.0 (indicating perfect dispersion) and +1.0 (perfect correlation). A zero value indicates a random spatial pattern.

So, sub-pixel mapping can be formulated a multi-objective optimal problem, while maximizing the spatial dependence index (SDI) and Moran's I, simultaneity using the following equations from equation (1).

$$
\begin{gathered}
\operatorname{Max} Y=f(x)=\left(f_{1}(X), f_{2}(X)\right) \\
\text { s.t. } \sum_{i=1}^{C} x_{i j}=1 \quad \sum_{j=1}^{D} x_{i j}=N C_{i}
\end{gathered}
$$

\section{MULTI-OBJECTIVE MEMETIC ALGORITHM}

\subsection{General framework of multi-objective memetic algorithm (MOMA)}

For a multi-objective optimization problem, $\max f(X)$, related definitions are as follows.

Definition 1 (Pareto dominance): For the arbitrary decision vector $X_{1}, X_{2} \in \Omega$, it is called that $X_{1}$ Pareto dominates $X_{2}$, namely $X_{1} \succ X_{2}$, if $f_{i}\left(X_{1}\right)>f_{i}\left(X_{2}\right)$; it is called that $X_{1}$ Pareto weakly dominates $X_{2}$, namely $X_{1} \succeq X_{2}$, if $f_{i}\left(X_{1}\right) \geq f_{i}\left(X_{2}\right), i=1,2, \ldots, m$.

Definition 2 (Pareto-optimal): For a decision vector $X^{*} \in \Omega$, if there not exist $X \in \Omega$ which satisfies $f_{i}(X) \geq f_{i}\left(X^{*}\right)$ and $f_{j}(X)<f_{j}\left(X^{*}\right)$,where $\mathrm{j}$ is an arbitrary value in the range $[1$, $\mathrm{m}]$, it is called that $X^{*}$ is Pareto-optimal solution or Pareto nondominated solution.

Definition 3 (Pareto-optimal front): For a Pareto-optimal solution set $\left\{X^{*}\right\}$, it is called that $P F$ is Pareto-optimal front if $P F=\left\{f(X)=\left(f_{1}(X), f_{2}(X), \cdots, f_{m}(X)\right) \mid X \in\left\{X^{*}\right\}\right\}$.

To solve the multi-objective problem, Memetic algorithm (MA) is utilized as a form of population-based Evolutionary algorithms hybridized with individual learning procedure that are capable of performing local refinements. Without loss of generality, the framework of multi-objective memetic algorithms (MOMAs) can be summarized by Figure 2 .

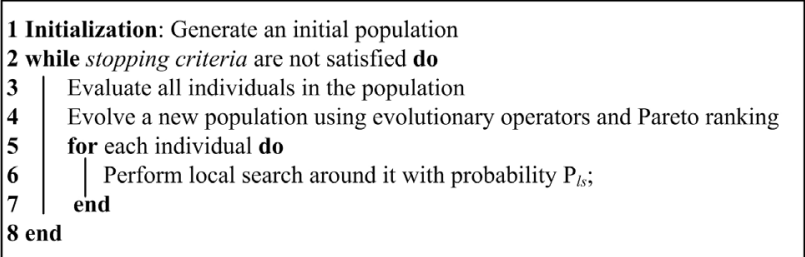

Figure 2 General framework for MOMAs
As shown in Figure 2, one major difference between MAs and conventional EAs is that the local search operator is added in addition to the evolutionary operators. Hence, the success of MAs is largely due to the appropriate adoption of local search operators. Unlike the evolutionary operators, which are usually very general and applicable to various problems, MOMAs provide the general framework, which the global search and the local search operators are usually expected to incorporate some domain specific heuristics, so that MAs can balance well between generality and problem specificity. In this paper, artificial immune algorithm is selected as the global search algorithm.

\subsection{Artificial immune algorithms for global search}

De Castro and Von zuben developed the Clonal Selection Algorithm on the basis of clonal selection theory of the immune system (De Castro and Von zuben, 2002). It was proved that can perform pattern recognition and adapt to solve multi-modal optimization tasks. The CLONALG algorithm can be described as follows:

Step 1: Randomly initialize a population of individual (M);

Step 2: For each pattern of $P$, present it to the population $M$ and determine its affinity with each element of the population $\mathrm{M}$;

Step 3: Select $n$ of the best highest affinity elements of $M$ and generate copies of these individuals proportionally to their affinity with the antigen. The higher the affinity, the higher the number of copies, and vice-versa;

Step 4: Mutate all these copies with a rate proportional to their affinity with the input pattern: the higher the affinity, the smaller the mutation rate;

Step 5: Add these mutated individuals to the population $M$ and reselect $\mathrm{m}$ of these maturated individuals to be kept as memories of the systems;

Step 6: Repeat steps 2 to 5 until a certain criterion is met.

\section{ADAPTIVE MULTI-OBJECTIVE SUB-PIXEL MAPPING FRAMEWORK BASED ON MEMETIC ALGORITHM FOR HYPERSPECTRAL REMOTE SENSING IMAGERY}

Based on the multi-objective sub-pixel mapping problem, the adaptive multi-objective sub-pixel mapping framework based on memetic algorithm, namely MSMF, is proposed. In contrast to the previous single-objective optimization for sub-pixel mapping, such as genetic sub-pixel mapping algorithm (GASM) (Mertens et al., 2003), MSMF has no single global solution, and it is often necessary to determine a set of points that all fit a predetermined definition for an optimum using the concept of Pareto optimality (Coello et al., 2007). As shown in Figure 3, MSMF provides the framework, and the evolutionary algorithms and local search may be selected using different algorithms according to the real problem. In this paper, clonal selection algorithm and random swapping algorithm are selected. 


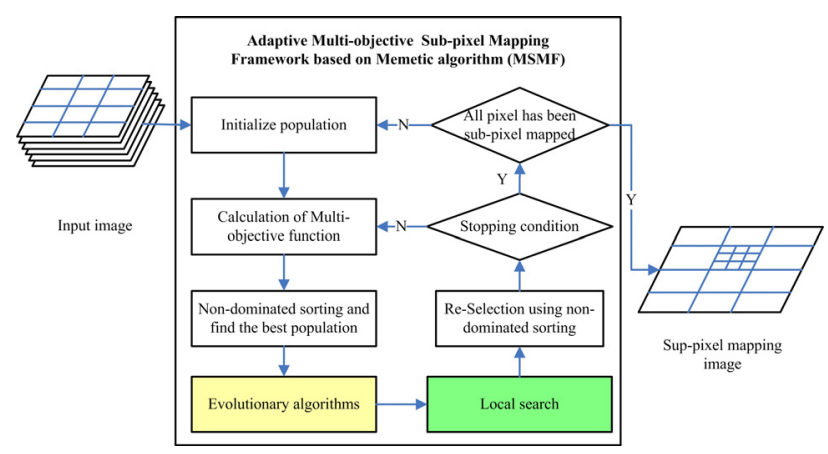

Figure 3 Adaptive multi-objective sub-pixel mapping framewok based on memetic algorithm (MSMF)

The MSMF is completed according to the following steps.

\subsection{Initialization}

In the initial step, each individual $a b_{t}$ in MSMF represents the possible sub-pixel configuration of the pixel and is directly described by a string consisting of integer numbers, which the length of each antibody string is equal to the number of subpixels. $a b_{t}=\left\{b_{1}, b_{2}, \cdots, b_{P}\right\}$, where the value of each bit in the string $a b_{t}$ represents the class of each sub-pixel. Because the number of sub-pixels for each class $N C_{i}$ has been calculated by fraction images in adavance, the algorithm should satisfy the limition of equation (3). The initial process is as follows: for each class $i$, selecting $N C_{i}$ sub-pixels using the following equation until all sub-pixels have been assigned to the land cover class

$$
\begin{aligned}
& j=\operatorname{Irandom}(1, D) \\
& b_{j}=i \quad i=1,2, \cdots, C
\end{aligned}
$$

where $D$ is the number of sub-pixels, C is the number of land cover classes, and funciton $\operatorname{Irandom}(1, D)$ returns a random integer value with the range $[1, D]$. After initialization, the simulation of the clonal selection process begins.

\subsection{Calculation of affinity}

According to the initial antibody population, the affinity of all $M A b$ 's in the antibody population $A B$ are calculated using the criterion function $f_{1}(a b)$ and $f_{2}(a b)$ using equation (3) and (5).

\subsection{Selection using non-dominated sorting}

(1)Identify the non-dominated solutions in the population and store them in a set $\mathrm{Pf}_{k}$ representing Pareto Front according to the definition 1-3, setting front count $\mathrm{k}=1$;

(2)Remove the non-dominated individuals from the temporary.

(3) $k=k+1$, identify the non-dominated solutions in the remaining population and store them in a set $\mathrm{Pf}_{k}$

(4)Repeat (3) and (4) until all individuals are separated into different fronts.

' $n$ ' higher affinity antibodies are selected to compose a new set $A B_{\{n\}}$ of high-affinity antibodies according to the order of the set of Pareto Front. The memory cell set is selected using Pareto Front 1.

\subsection{Clone}

After receiving antibody individuals closer to the solution, the next generation should mainly be derived from the betterfitting individuals. Thus, the $n$ selected $a b$ 's are cloned based on their antigenic affinities, generating the clone set $C$. The total number of clones-generated $N_{c}$ is defined as follows:

$$
N_{c}=\sum_{i=1}^{n} \operatorname{round}(\beta \cdot M)
$$

where $\beta$ is a multiplication factor, $M$ is the total number of antibodies, round $(\cdot)$ is the operator that rounds its argument.

\subsection{Mutation}

Provide each $a b$ in the clone set $C$ with the opportunity to produce mutated offspring $C^{*}$. The higher the affinity, the smaller the mutation rate. The mutation process utilizes the non-uniform mutation operator, and exchanges the position of the sub-pixels. To improve the intelligence of MSMF, the mutation rate of each cloned antibody, pm, is adaptively determined according to its affinity.

\subsection{Local search}

There is always a possibility of stagnation in MSMF. To move away from the point of stagnation, a feasible operation is a neighborhood or local search, which can be applied to a solution to find a more feasible solution in the local neighborhood. In this paper, the values in the individual indexed by $i$ and $j$ are then swapped. The objective function of the new individual is calculated, and only if there is an improvement is the new solution accepted. In addition, the pixel-swapping algorithm is also utilized in the local search.

\subsection{Re-calculation of affinity}

Calculate the affinity $f_{1}^{*}(a b)$ and $f_{2}^{*}(a b)$ using equation (9) and (5) of the matured clones $C^{*}$.

\subsection{Re-selection}

After cloning and mutation, the new offspring combines $\mathrm{C}^{*}$ with the parent population to form a temporary population. The new population $\mathrm{AB}$ is obtained by the non-dominated sorting and selection (See also 4.3).

\subsection{Updating the memory cell set}

Select the solutions in Pareto front 1 to form memory set.

\subsection{Stopping condition}

If the generation, $G$, does not meet the maximum generation number, $G_{\max }$, go to step 4.3. Otherwise, output the best individuals as the sub-pixels of the pixel $x_{i j}$. The above process is repeated from Step 4.1 until the sub-pixels of all pixels in the fraction image are located. Finally, the proposed algorithm outputs the sub-pixel mapping image.

\section{EXPERIMENTS AND ANALYSIS}

\subsection{Experiment 1-HJ-1A hyperspectral imagery}


The synthetic real hyperspectral remote sensing image based on Chinese HJ-1A image with 115 bands $(0.45-0.95 \mu \mathrm{m})$ was used to test the performance of the proposed sub-pixel mapping algorithm by comparing it with the DNSM, SASM, BPSM, and GASM algorithms. The HJ-1A satellite is a small Chinese environmental satellite. The HJ-1A image $(256 \times 256)$, shown in Figure 4 (a), was acquired on August 19, 2009 (Path: 01, Raw: 79) and was used as the original image for the experimental results. The study site is located in Hanchuan city, Hubei province, central China, and its surrounding area. Four landcover classes, i.e., city, agricultural land, water, and vegetation, characterize this image. Figure 4 (b) shows the hard classification result for the Hanchuan HJ-1A image obtained by a support vector machine (SVM), an effective classifier for hyperspectral remote sensing image, in ENVI software. The fraction images of the four classes were obtained, as shown in Figure 4 (c), by resampling with a scale of 2 . Figure $4(d)-(h)$ show the sub-pixel mapping results obtained by using DNSM, SASM, BPSM, GASM, and the proposed algorithm MSMF. To quantitatively evaluate the sub-pixel mapping accuracy, the original hard classification result, as the real sub-pixel mapping result, was used to test the performance of the five algorithms. Two small images of S1 and S2 areas were zoomed and are shown in Figure 4 (i) to evaluate all the sub-pixel mapping algorithms from the visual results.

As shown in Figure 4 (d)-(i), DNSM and BPSM did not provide satisfactory visual results, as there is blurring and many structural data are lost. In addition, the sawtooth phenomenon can be observed in the BPSM result. By contrast, SASM, GASM, and MSMF have obtained better visual results, being smoother and with most classes' structural information preserved. A quantitative comparison of the five algorithms is shown in Table 1 using the overall accuracy (OA), Kappa coefficient (Kappa). In addition, an adjusted confusion matrix was utilized, which it is calculated only for mixed pixels. Based on the adjusted confusion matrix, we adjust OA (OA*), Kappa coefficient (Kappa*), the average of the producer's accuracy (APA), and the average of the user's accuracy (AUA). As with the visual results, the sub-pixel mapping accuracy of DNSM and BPSM are lower than that of the other algorithms. For instance, one can see from Table I that the average producer's accuracies of DNSM and BPSM are equal to $61.34 \%$ and $68.72 \%$, respectively. SASM and GASM improves the subpixel mapping accuracy and locates the sub-pixels' position better than the aforementioned algorithms, resulting in a producer's accuracy of $72.89 \%$ and $72.61 \%$. It was MSMF, however, that obtained the highest overall accuracy, Kappa coefficient, adjusted overall accuracy, and adjusted Kappa coefficient, which are $79.97 \%, 0.7282,74.22 \%$, and 0.6504 , respectively. One reason may be that MSMF considers not only the spatial dependence between pixels but also within pixels. Experimental results demonstrate that the proposed approach outperform the other four algorithms for this data set.

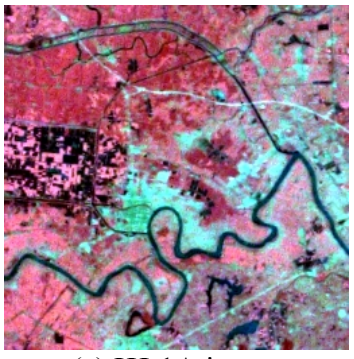

(a) HJ-1A image

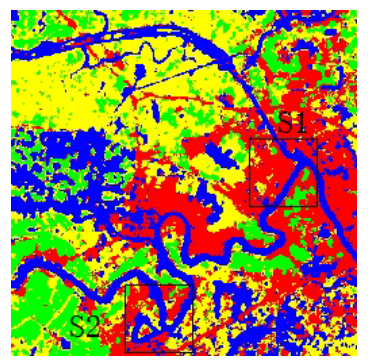

(b) Classification result

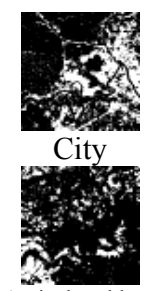

Agricultural land

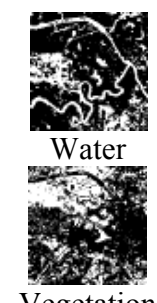

(c) The fraction images

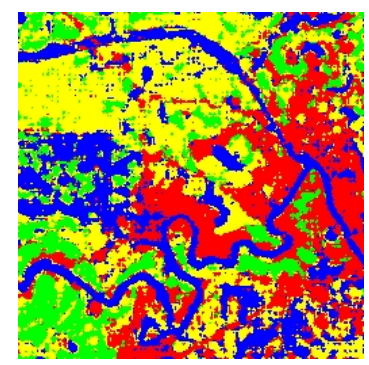

(e) SASM

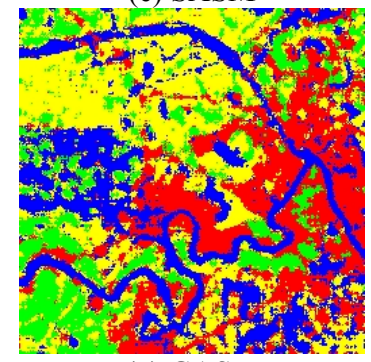

(g) GASM
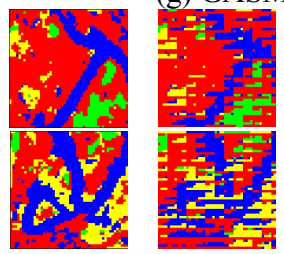

Zoom (d)

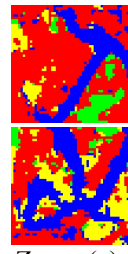

Zoom (e)

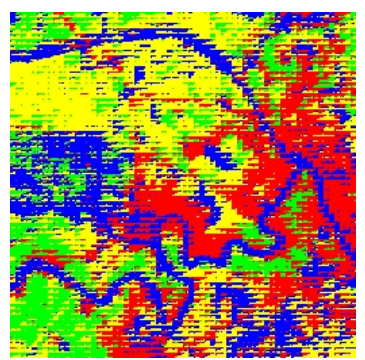

(d) DNSM

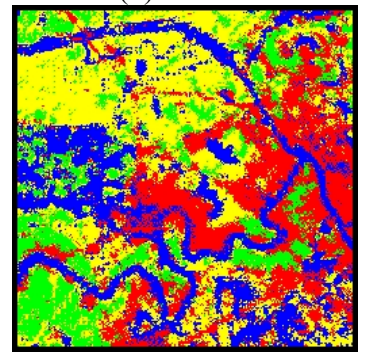

(f) BPSM

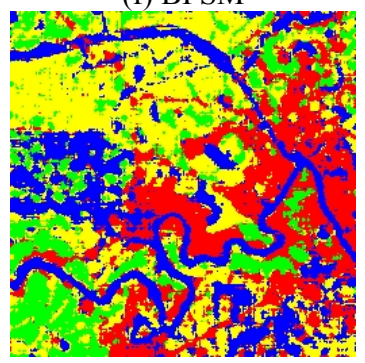

(h) MSMF

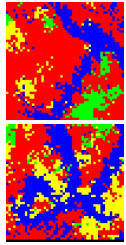

Zoom (f)

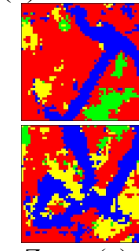

Zoom (g) (i) Zoom images for all sub-pixel mapping results

City Agricultural land Water Vegetation

Figure 4. The sub-pixel mapping results in the experiment 1

\begin{tabular}{|c|c|c|c|c|c|}
\hline Scale $=4$ & DNSM & SASM & BPSM & GASM & MSMF \\
\hline OA $(\%)$ & 70.11 & 78.88 & 75.80 & 78.69 & $\mathbf{7 9 . 9 7}$ \\
\hline Kappa & 0.5944 & 0.7134 & 0.6713 & 0.7109 & $\mathbf{0 . 7 2 8 2}$ \\
\hline OA $^{*}(\%)$ & 61.53 & 72.82 & 68.80 & 72.57 & $\mathbf{7 4 . 2 2}$ \\
\hline Kappa & 0.4782 & 0.6313 & 0.5766 & 0.6280 & $\mathbf{0 . 6 5 0 4}$ \\
\hline APA(\%) & 61.34 & 72.89 & 68.72 & 72.61 & $\mathbf{7 4 . 3 1}$ \\
\hline AUA(\%) & 61.34 & 72.89 & 68.72 & 72.61 & $\mathbf{7 4 . 3 1}$ \\
\hline
\end{tabular}

Table 1. Comparison of five sub-pixel mapping algorithm performances for the synthetic HJ-1A hyperspectral remote sensing image

\subsection{Experiment 2-Washington D. C. HYDICE imagery}

In experiment 2, a part of Hyperspectral Digital Imagery Collection Experiment (HYDICE) airborne hyperspectral data over the Washington, D. C. Mall with 192 bands was used, which it comprised 300 lines and 240 columns as shown in Figure 5 (a). Figure 5 (b) shows the reference image classified by the Support Vector Machine (SVM) method, as the truth data. The observed image was expected to fall into six classes: roof, road, trail, grass, shadow, and tree. Figure 5 (d)-(g) show 
the sub-pixel mapping results obtained by using DNSM, SASM, BPSM, GASM, and the proposed algorithm MSMF.

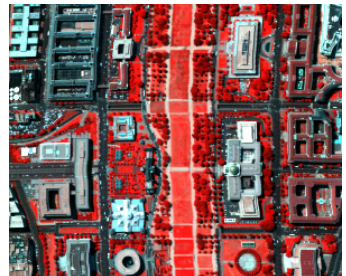

(a) Washington D.C. Image

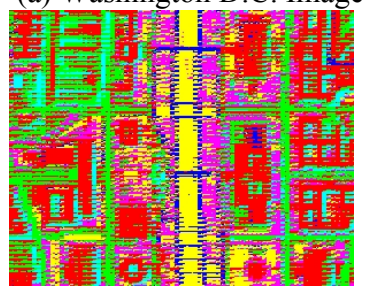

(c) DNSM

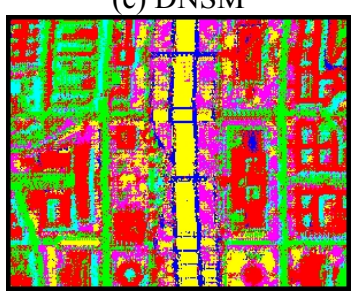

(e) BPSM

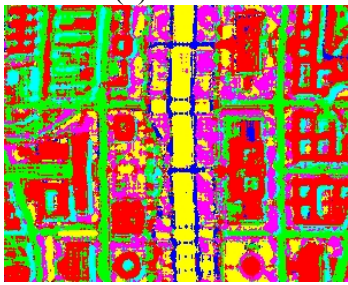

(g) MSMF

Figure 5. The sub-pixel mapping results in the experiment 2

\begin{tabular}{|c|c|c|c|c|c|}
\hline Scale $=4$ & DNSM & SASM & BPSM & GASM & MSMF \\
\hline OA $(\%)$ & 64.82 & 74.05 & 70.08 & 73.21 & $\mathbf{7 5 . 2 2}$ \\
\hline Kappa & 0.5490 & 0.6673 & 0.6171 & 0.6565 & $\mathbf{0 . 6 8 2 4}$ \\
\hline OA $^{*}(\%)$ & 55.28 & 67.01 & 61.93 & 65.94 & $\mathbf{6 8 . 5 0}$ \\
\hline Kappa $^{*}$ & 0.4372 & 0.5849 & 0.5217 & 0.5714 & $\mathbf{0 . 6 0 3 7}$ \\
\hline
\end{tabular}

Table 2. Comparison of five sub-pixel mapping algorithm performances for the synthetic Washington D. C. hyperspectral remote sensing imagery

As shown in Figure 5 and Table 2, MSMF obtains smoother results with most classes' structural information preserved and has the highest overall accuracy, Kappa coefficient, adjusted overall accuracy, and adjusted Kappa coefficient, which are $75.22 \%, 0.6824,68.50 \%$, and 0.6037 , respectively. Compared to conventional EAs, for example, genetic algorithm, there are two possible key issues for the success of MSMF. One is an appropriate balance between global and local search, and other is a cost effective coordination of local search. Based on the above results, MSMF can obtain better sub-pixel mapping results for the complex hyperspectral remote sensing image with more classes and higher spatial resolution.

\section{CONCLUSION}

Based on memetic algorithm, this paper proposed an adaptive multi-objective sub-pixel mapping framework, namely MSMF, for hyperspectral remote sensing images. The proposed MSMF defines two objective function to describe the spatial dependence between pixels and sub-pixels. The sub-pixel mapping problem is transformed to a multi-objective problem. MSMF utilizes the memetic algorithm to solve this problem by combining the global search and local search. The experimental result shows that MSMF algorithm consistently outperforms the previous sub-pixel mapping algorithms, and hence provides an effective method for remote sensing image sub-pixel mapping.

\section{ACKNOWLEDGEMENTS}

This work was supported by National Basic Research Program of China (973 Program) under Grant No. 2011CB707105, the National Natural Science Foundation of China under Grant Nos. 40901213, 40930532, A Foundation for the Author of National Excellent Doctoral Dissertation of P.R. China (FANEDD) under Grant No. 201052, Program for New Century Excellent Talents in University under Grant No.NECT-10-0624, and the Fundamental Research Funds for the Central Universities under Grant No.3103006.

\section{REFERENCES}

Atkinson, P. M., 1997. Mapping sub-pixel boundaries from remotely sensed images. in Innovations in GIS 4, Z. Kemp, Ed., pp. $166-180$.

Chang, C.-I., 2003, Hyperspectral Imaging: Spectral Detection and Classification. New York: Plenum.

Coello, C. A. C., Lamont, G. B., and Veldhuizen, D. A. V., 2007. Evolutionary algorithms for solving multi-objective problems (Second Edition) vol. 5: Springer-Verlag New York Inc.

Dasgupta, D., Yu, S. and Nino, F., 2011. Recent Advances in Artificial Immune Systems: Models and Applications. Applied Soft Computing, 11(2), pp. 1574-1587.

De Castro, L. N., and F. J. Von Zuben, 2002. Learning and optimization using the clonal selection principle. IEEE Transactions on Evolutionary Computation, 6, pp. 239-250.

Mertens, K. C., Baets, B. D., Verbeke, L. P. C., and Wulf, R. R. D., 2006. A sub-pixel mapping algorithm based on subpixel/pixel spatial attraction models. International Journal of Remote Sensing, 27(15), pp. 3293-3310.

Mertens, K. C., Verbeke, L. P. C., Westra, T., and Wulf, R. R. D., 2003. Using genetic algorithms in sub-pixel mapping. International Journal of Remote sensing, 24(21), pp. 42414247.

Moran, P. A. P., 1950. Notes on Continuous Stochastic Phenomena. Biometrika, 37 (1), pp. 17-23.

Ong, Y.-S., Lim, M., and Chen, X., 2010. Memetic Computation-Past, Present \& Future. IEEE Computational Intelligence Magazine, 5(2), pp. 24-31.

Verhoeye, J. and Wulf, R. De., 2002. Land cover mapping at sub-pixel scales using linear optimization techniques. Remote Sensing of Environment, 79(1), pp. 96-104.

Zhang, L., Wu, K., Zhong, Y., and Li, P., 2008. A new subpixel mapping algorithm based on a BP neural network with an observation model. Neurocomputing, 71(10-12), pp. 20622054.

Zhong, Y. and Zhang, L., 2012. Remote sensing image subpixel mapping based on adaptive differential evolution. IEEE Transactions on Systems, Man, and Cybernetics Part B, DOI : 10.1109/TSMCB.2012.2189561. 